\title{
Health status, outcome, and attributability: is a red rose red in the dark?
}

\author{
John Shanks, Alison Frater
}

Dabblers in philosophy and logic may recall deriving intellectual enjoyment from mind teasers such as: "If a tree falls in the forest and there is no one to hear it, does it make a noise? Is a red rose red in the dark?"

The Department of Health clearly hopes that measurement of health outcomes will form a central part of the drive to improve the efficiency and effectiveness of the NHS - but what constitutes a measure of health outcome? Is change in health status a measure of outcome if we don't know to what extent it reflects the impact of health care as opposed to other factors? Or must we wait until research has fully disentangled the contribution of treatment before we bestow the description "outcome" on the measure in question? A subsidiary problem concerns the nature of outcome measurement and to whom its findings are most appropriately addressed - is the activity audit or research and are the results of interest to clinicians, managers, or service users?

We offer for discussion the view that measurement of outcome as change in health status can be valuable even before we know precisely the contribution of health care to observed changes. We propose a terminology which reflects this. We suggest that outcome measurement has a legitimate place in both audit and research. We put forward the view that health service users and managers, as well as clinicians, stand to benefit from the more widespread measurement of health outcomes.

\section{Influences on health}

Making sense of outcomes in relation to health takes us back to defining health and where it comes from. The task of definition is famously difficult and has prompted attempts ranging from the modest to the encyclopaedic. ${ }^{12}$ Many of the currently influential definitions of health take a broad view which encompasses both wellbeing and functional ability in several different areas of life. None claims that all of life is health, and so all imply the existence of non-health aspects of status and functioning which, although they may have an effect on health, are not themselves defined as health (for example, economic status, artistic ability, and spiritual awareness). Health is generally agreed to be subject to the influence of a wide range of social and environmental forces. The effect of health care is often an indeterminate, but probably small, drop in that ocean. It is rare to be able unequivocally to ascribe observed changes in health status solely to health care provided. Similarly, the effects of health care can go well beyond a change in health status to produce an impact in other areas of life - on personal financial circumstances, for example. All of this implies that we will sometimes wish to distinguish between those aspects of health status which are the result of health care and those which are not and between those effects of health care which are manifest as a change in health status and those which are evident in some other way. If our concept of health outcomes is to be consistent with our view of health and what influences it, then it should accommodate these same distinctions.

\section{Partial and variable attributability}

The drive towards measuring outcomes originated among those with an interest in assessing the quality and effectiveness of health services. Not surprisingly, then, there is one view that it is axiomatic that any valid measure of health outcome must be attributable to health care. ${ }^{3}$ In reality, things are more complicated because attributability is a matter of degree rather than an all or none phenomenon and because we usually only partly understand the causal factors involved and the place of health care among these. Donabedian's early writings on outcomes refer to the necessary assumption "that good results are brought about . . . at least to a significant degree, by good care." 4 Different health outcomes are attributable to health care to differing extents. The relative contribution of factors other than health care may also vary. This variation in attributability applies both to populations and to individuals. Those who swim with the tide usually achieve higher speeds than those who must fight the current. When the real price of alcohol is falling, even the most effective campaign to get people to cut down their drinking will be likely to have less impact on behaviour than when prices are stable or rising. The same observed fall in blood pressure in two patients receiving the same antihypertensive drug may be almost entirely due to the treatment in one who is a passive recipient of care but largely due to different factors in the other who has recently decided to give up a stressful job and has taken 
up meditation. So a workable definition of outcome must be able to encompass partial and variable attributability of health status to health care at both population and individual level.

\section{A proposed terminology}

Before the health evaluators colonised it, an "outcome" merely meant an end point or result..$^{5}$ The notion of an outcome as a result implies two links in the chain: the cause and the resulting effect. It is possible to specify either one or both. Our knowledge of the relevant causal factors is usually incomplete, and there are degrees of attributability to each factor. This suggests a conceptual scheme and a terminology which can accommodate degrees of attributability to causal factors which we only partly understand. Within this scheme, an outcome (measured as a change in some variable) is meaningful even if, for the time being, we can measure only the change itself and not the causes responsible for it. When we become able to identify the cause or causes and subsequently measure their contribution to the change, the measure acquires an additional layer of meaning.

\section{Recognising types of outcome}

The conceptual scheme proposed in the box recognises the existence of different types of outcome appropriate for different purposes. All of the terms are applicable either at individual or at population level.

The term outcome is reserved as a general descriptor for use in discussion with practitioners other than health practitioners,

\section{Levels of outcome: a proposed classification scheme}

Outcome

A result. May be evident in any sphere of life such as health, social, or economic circumstances. Neither the cause nor the nature of the effect is specified.

Health outcome

An effect manifest as change in health status. The causal factors involved and their relative contributions are left unspecified and may be unknown.

Health care outcome

A result which is attributable and responsive to health care. The causal connection to health care has been established and its strength of contribution at least partly identified. The area in which the effect is evident is left unspecified - it could be a change in health status but could equally be a change in social or economic circumstances, for example.

Health outcome of health care

A result evident in terms of health status which is attributable and responsive to health care. It is therefore a subset of "health care outcome." Both the cause and the effect are known and specified. The causal connection to health care has been established and its strength of contribution at least partly identified. who are also interested in investigating cause and effect connections.

The concept of a health outcome is relevant to clinicians who need to monitor the progress of individual patients under their care while recognising that the changes they see are multifactorial, with an as yet indeterminate contribution from health care. Patients and their carers may value a prognosis even though gaps remain in professional understanding of the forces which shape it. At the population level public health practitioners need to describe the changing health status of a population, which may be subject to a complex and unspecified mixture of changing social, economic, and environmental forces.

The notion of the health care outcome is of interest to health service managers, researchers, and clinicians seeking to distinguish the impact of health care from other factors. The need for efficient deployment of scare resources and accountability for their use exerts a pressure in this direction. Patients have choices to make between alternative treatment options, and they require information on what results they can expect from each. ${ }^{6}$ Providing users with information about the risks and benefits of care, outcome probabilities, and alternative interventions shifts the locus of decisions about trade offs more towards the patient.

A health outcome of health care is relevant to the same audience as above but restricts the focus of interest to changes in health status alone. Such a distinction may be necessary in order to place achievements of health care in the broader context of human life or to make explicit distinctions between the effects of health care on different areas of functioning. A person who has had treatment of known efficacy for a severe mental illness may have made a good recovery by all measured parameters of mental health but may nevertheless fail in attempts to regain employment or a valued place in the community. The observation of a discrepancy between a good health outcome from health care and a compromised way of life may help identify areas of functioning not included in the assessment of recovery which are impaired and which are crucial limiting factors. Or it may direct attention and efforts outwards towards broader societal factors, such as the popular opinion of those who have suffered mental illness.

\section{Attributability: handling uncertainty, extending knowledge}

Measurement of an effect whose causes remain partly unidentified can allow observation of progress and trends in health. Is this of any value? Systematic measurement of individual health status in clinical practice is conceptually equivalent to asking the question: "How are things?" The question may be directed to the individual concerned (self assessed health status) or asked of one person about another (objective health status). A cross sectional study permits comparison of the answers from many different people at one 
point in time, while a longitudinal inquiry allows monitoring of progress of the same people over time. The fact that we all inquire so assiduously after the health of others in both social and clinical settings suggests an appreciation that knowing how someone is has a value even before we know why. Even before we knew that thunder is a product of lightning it was possible and worthwhile to note that the two phenomena usually occur together, that they are more likely in warm weather, and that they frequently predict imminent heavy rain. The collection of aggregated information on patients' health states can establish standards for comparison, improve prognostic accuracy and generate causal hypotheses for testing. There are enough samples of bad outcome following good process to make this worth exploring. The UK Clearing House for Information on the Assessment of Health Service Outcomes has adopted a broad definition of outcomes which includes "patient centred outcomes" such as quality of life measures, ${ }^{7}$ even though it will often be unclear at the time of measurement to what extent these are influenced by treatment.

Of course, as good natural scientists we have an interest in asking "why?" when we observe a particularly good or bad result. We are instinctively drawn towards testing hypotheses on the attributability of the outcomes which we measure. The most appropriate method of doing this depends on the nature of the question and the resources available to pursue it. ${ }^{8}$ Where a randomised controlled trial is possible it may be the most powerful method. However, the opportunity to extend our knowledge of cause and effect need not be conditional on the ability to mount such a trial. The alternative method of observational inference - that is, testing observed associations between process and outcome for causal links in accordance with well established rules of scientific evidence ${ }^{9}-$ offers a complementary approach which can coexist with randomised controlled trials. ${ }^{10}$

Even for those outcome measures for which the contribution of health care is known and quantified, there is the problem of dealing with the existence of multiple perspectives on the same outcome. The evidence suggests that the patient's view of personal wellbeing often differs from the hospital clinician's objective assessment ${ }^{11-13}$ and that patients tend to be more risk-averse than clinicians in selecting the favoured treatment option. ${ }^{14}$ It may be quite a challenge to reconcile very different points of view on the same situation, but the advantage is to make explicit those differences of opinion which were already present and capable of exerting an effect - on compliance, for example - even while unexpressed. The aim would be to move towards a negotiated and agreed position on preferred management which bridges clinicians' and patients' perspectives. Where, for instance, the assessment of the patient, the clinician, and the informal carer differ, it may be necessary to agree some process of weighting or trade off between them.

\section{Audit or research?}

The government's white paper Working for Patients explicitly included outcomes within the remit of medical audit. ${ }^{15}$ The central contribution of research in charting the effects of service interventions goes without saying. The measurement of outcomes thus has a place both in audit and in research. Extending audit beyond its current preoccupation with process into the arena of outcomes offers the opportunity to assess the benefits of health care and generate testable hypotheses on causal connections between process and outcome, including, perhaps, hitherto absent information on patient preference for outcome. Comparison of outcomes between one clinician and another or one hospital and another generates hypotheses for research and identifies areas which merit further local investigation. Standards for expected outcomes can be derived from research evidence. Comparison between these standards and the actual outcomes achieved in practice constitutes an audit which can inform efforts to improve quality. These comparisons will need to take account of factors such as case mix and severity. Standards can also be set between clinicians and patients on the level of health status which it is feasible to achieve. Subsequent audit may allow the clinician to review for a selection of patients to what extent the offered benefit of therapeutic intervention the achievement of the desired level and pattern of health status - has been fulfilled. As further information becomes available on norms for health status in the general population and for specific diagnostic groups, more detailed targets can be agreed. Patients provided with information on the outcome probabilities of alternative interventions are enabled to choose a course of action (for example, whether to have surgery or not) on the basis of their own perception of the risks and benefits of treatment, their tolerance of symptoms, and their preference for the possible outcomes, rather than as the passive recipient of clinical advice. The uncertainty which is a ubiquitous problem in clinical decision making then also becomes the provenance of the consultation between clinician and patient. Collecting information on patient preference for outcomes is a key part of the effectiveness research undertaken by the Patient Outcomes Research Team established in the United States by the Agency for Health Care Policy Research (AHCPR). ${ }^{16}$ This body of work links clinical practice, audit, and research, and it seeks to inform clinical trials, to shed light on variations in practice, and to fix patients' values firmly at the centre of both audit and health care evaluation.

\section{Conclusions}

Research methodology must continue to produce reliable and well validated measures of the outcome of health care and can define standards of outcome which can be expected from the application of specific interventions. Standards thus defined then become the basis for subsequent audit of clinical practice. 
The shared requirement for measurement of outcome should help draw attention to the common spirit of scientific inquiry which unites audit and research as being more important than the differences in emphasis and style which divide them. ${ }^{17} 18$ Health service managers intent on purchasing for maximum health gain need information from the sort of robust and attributable measures of outcome which the combined forces of research and audit can provide. The difficult choices around treatment options which clinicians and patients share are enlightened by clear information on the associated probable outcomes. The growing interest in developing formal guidelines on best clinical practice offers one mechanism by which evidence on health care outcomes can be fed back to clinicians to improve their practice. ${ }^{19}$ Not only clinicians but also patients, managers, and purchasers of health services could benefit directly from access to appropriate outcome-based clinical guidelines by refining their ability to judge the quality of care on offer. We may finally be in a position to answer affirmatively Schroeder's question: "Outcome assessment 70 years later: are we ready?" 20

We thank Dr Geoff Shepherd, whose comments provided the starting point for this paper, and Dr Azim Lakhani, Kieran starting point for this paper, and Dr Azim Lakhani, Kieran
Walshe, Dr Jenny Bennett, and the unknown referees for their encouragement and advice.

1 Goldsmith SB. The status of health status indicators. Health Serv Rep 1972;87:212-20.
2 Goldsmith SB. A re-evaluation of health status indicators. Health Serv Rep 1973;88:937-41.

3 Hopkins A, ed. Measuring the quality of medical care. London: Royal College of Physicians, 1990.

4 Donabedian A, ed A guide to medical care administration. Vol 2. New York: American Public Health Association, 1969:3.

5 Oxford English dictionary. 2nd ed. Oxford: Oxford University Press, 1989.

6 Wennberg JE, Bunker JP, Barnes B. The need for assessing the outcome of common medical practices. Ann Rev the outcome of common medic

7 Long AF, Bate L, Sheldon TA. Establishment of the UK clearing house for assessing health services outcomes. Quality in Health Care 1992;1:131-3.

8 Ellwood J. Causal relationships in medicine: a practical system for critical appraisal. Oxford: Oxford University Press, 1988.

9 Hill AB. The environment and disease. Association and causation. In: Fletcher RH, Fletcher SW, Wagner EH eds. Clinical epidemiology - the essentials. Baltimore: Williams and Wilkins, 1988:216-20.

10 Cross-design synthesis: a new strategy for studying medical outcomes? Lancet 1992;340:944-5.

11 Nerenz DR, Repasky DP, Whitehouse FW, Kahkonen DM. Ongoing assessment of health status in patients with Ongoing assessment of health status in patients
diabetes mellitus. Med Care 1992;30(suppl):112-24

12 Rubinstein LV, Schairer C, Wieland GD, Kane $R$ Systematic biases in functional status: effects of different data sources. $\mathcal{F}$ Gerontol $1984 ; 39: 686$.

13 Black N, Petticrew M, Ginzler M, Flood A, Smith J, Williams $\mathrm{G}$, et al. Do doctors and patients agree? Views of the outcomes of transurethral resection of the prostate Int $\mathcal{F}$ Technol Assess Health Care 1991;7:533-44.

14 McNeil BJ, Weichselbaum R, Pauker SG. Fallacy of the five year survival in lung cancer. $N$ Engl $f \mathrm{Med}$ 1978;299:1397-401.

15 Secretaries of State for Health, Wales, Northern Ireland and Scotland. Medical audit. Working paper 6. London: HMSO, 1989.

16 Raskin IE, Maklan CW. Medical treatment effectiveness research - a view from inside the Agency for Health Care Policy Research. Evaluation and the Health Care Professional 1991;14:161-85.

17 Smith R. Audit and research. BMF 1992;305:905-6.

17 Smith R. Audit and research. BMF 1992;305:905-6. Quality in Health Care 1993;2:1-2.

19 Haines A, Feder G. Guidance on guidelines. $B M \mathcal{F}$ 1992;305:785-6.

20 Schroeder RA. Outcome assessment 70 years later: are we ready? $N$ Engl $\mathcal{f}$ Med 1987;316:160-2. 